\title{
Robot Assisted Internal Mammary Artery Detection for Coronary Revascularisation Surgery
}

\author{
Florian A. Fröhlich*, Georg Passig*, Adrian Vazquez** and Gerd Hirzinger* \\ (*) German Aerospace Center (DLR) (**) ICCAS, University of Leipzig
}

\begin{abstract}
This paper presents a semi-automatic robotic system supporting a surgeon in the harvesting of the internal mammary artery (IMA) for an open chested intervention in coronary revascularisation surgery. The versatile surgical lightweight robot MIRO developed at DLR (German Aerospace Center) is used to detect and mark the path of the IMA at the inner side of the thoracic wall. The robot is equipped with a tool combining a Doppler ultrasonography (US) probe and a medical marker pen. The position of the IMA is extracted from the US-images to place the tool above the artery via visual servoing. Additionally, the robot moves the tool in direction of the artery to mark the location of the IMA on it's path. To achieve an ideal contact situation for US-imaging along the whole path the contact force between tissue and probe is controlled according to force measurements based on the internal torque sensors of the robot. The evaluation of the robotic system by an animal experiment shows that the system is capable of robustly detecting the IMA.
\end{abstract}

\section{INTRODUCTION}

In coronary revascularisation surgery or coronary bypass graft $(\mathrm{CABG})$ surgery the internal thoracic arteries are used to supply the regions of the heart beyond blocked coronary arteries with oxygen saturated blood. Depending on the diagnosis the left internal mammary artery (LIMA) or the right internal mammary artery (RIMA) are used. The intervention is called Vineberg operation [1]. It is essential that the artery is not injured during the operation. Commonly, the IMA is detected manually starting at the cranial end (pointing to the head) close to the subclavian artery. In a conventional open chested intervention a possible approach to detecting the IMA is palpation. In totally endoscopic coronary bypass (TECAB) interventions [2], [3] usually only visual feedback is available. Therefore, the artery is detected referring to anatomic landmarks [4]. Afterward the artery is iteratively dissected to the point of the bifurcation of the IMA. There are several techniques [5], [6] for endoscopic operations yet the surgeon still has to manually detect and dissect the artery. The process is time-consuming and takes between 30 and 60 minutes [7].

The objective of this work is the development of a semiautomatic robotic assistance system aiding the surgeon in detecting the IMA and reducing the time of the intervention. Robotic assisted approaches to detecting the IMA with a tactile sensor in an endoscopic intervention are published in [8]. However, for the presented system Doppler USimaging is used as it is already commonly available for preand postoperative diagnosis [9] and therefore the surgeons are accustomed to the deployment and interpretation of the data. US-imaging in combination with image processing can

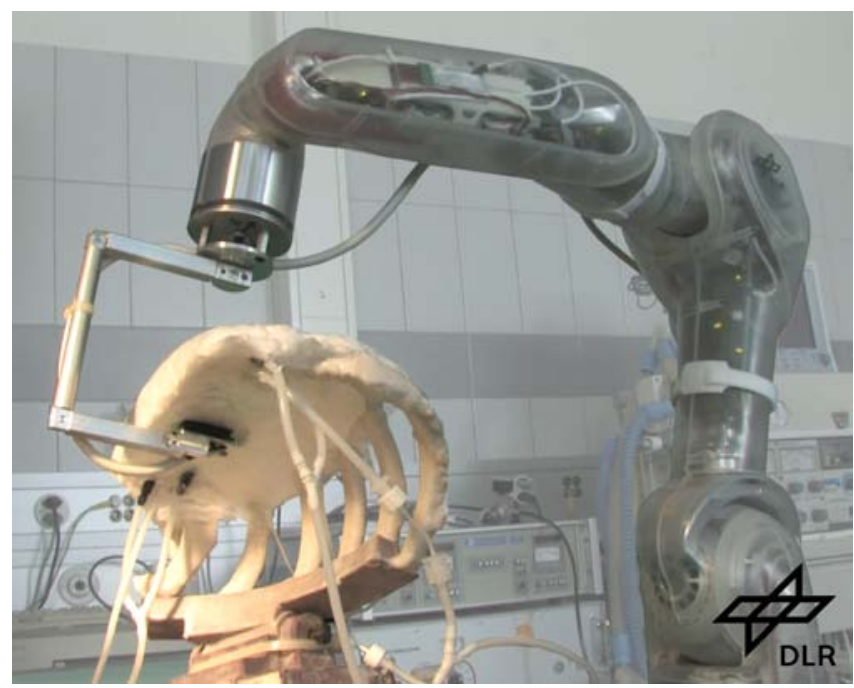

Fig. 1. MIRO robot with US-probe and patient phantom.

be used as measurement system for robotic applications as shown for e.g., needle guidance [10].

The proposed assistance system is aimed at detecting and marking the path of the IMA in an open chested intervention. The sensor selected for detecting the artery is a Doppler US-probe that is guided by the versatile surgical lightweight robot MIRO [11] depicted in Fig. 1. For the development of the system a patient phantom ${ }^{1}$ is used. The validation is performed in an animal trial. Although, the system is designed for conventional interventions, the chosen approach can also be applied to endoscopic interventions.

In the following the setup for semi-automatic robotic assistance in a conventional open chested IMA dissection is described. Sec. II outlines the technical setup of the system. The control schemes are introduced in Sec. III. Afterward the results of an animal trial used for the evaluation of the system are presented in Sec. IV. In Sec. V-B the results are briefly recapitulated and the modifications necessary for transferring the setup to an endoscopic intervention are mentioned.

\section{ROBOTIC SETUP}

The technical setup with the surgical lightweight robot MIRO carrying a c-shaped tool is depicted in Fig. 1. The USprobe is fixed to the lower bracket of the tool. The patient phantom is a replicate of a human rib cage with artificial tissue that has US-properties comparable to human tissue.

\footnotetext{
${ }^{1}$ Developed by ICCAS, University of Leipzig
} 
The embedded tubes are flooded with a fluid containing particles and are connected to a controlled pump system that simulates physiological pulsating blood flow. The phantom is used for adjusting the controller parameters and testing the usability of the system.

The components of the system that are used in experiments presented in Sec. IV are described in more detail in the following.

\section{A. MIRO Lightweight Robot}

The MIRO robot is a versatile surgical lightweight robot [11] designed for minimally invasive as well as conventional open interventions where robotic aid is helpful. The MIRO robot is composed of seven joints with serial kinematics, comparable to the human arm. The kinematics is redundant. The robot control supports different modes. In position controlled mode the MIRO robot is controlled by a full-state feedback control for flexible and coupled joints [12]. The controlled states are the motor positions and joint torques, which are both directly measured as well as the derivatives of both states. The additional disturbance observer uses the joint torque measurements to reduce friction in the motor and the gear boxes [13]. In the impedance controlled mode a Cartesian impedance controller [14], [15] is applied.

\section{B. Ultrasonic Probe}

The US-device used for the system setup is a General Electrics Vivid 7 ultrasound scanner with an i13L probe. The device provides video output signals in S-Video, S-VGA and composite video format. The probe is an electronically steered linear ultrasound array transducer operating in a frequency range of $5.3-14.0 \mathrm{MHz}^{2}$. The size of the contact surface of the probe is about $25 \mathrm{~mm} \times 5 \mathrm{~mm}$.

\section{Probe Mounting}

The c-shaped probe mounting tool depicted in Fig. 2 is connected to the robot by a magnetic coupling attached to the upper bracket. The US-probe is fixed to a socket at the lower bracket of the tool. The length of the brackets is about $7.5 \mathrm{~cm}$ to ensure the accessibility of any artery location in a reasonable range.

The coordinate system depicted in Fig. 2 is the tool frame. The $\mathrm{x}$-axis and the $\mathrm{z}$-axis are aligned to the axes of the USimaging-plane, the y-axis is the normal direction. The control schemes described in Sec. III refer to this frame.

The marker is composed of the tip of a medical pen. It is located slightly off the center of the US-probe in negative $\mathrm{x}$-direction. The line that is drawn then directly indicates a secure path for an incision that is parallel to the artery.

\footnotetext{
${ }^{2}$ The upper frequency limit is dependent of the ultrasound unit and software version (SV). The frequency values presented correspond to GE ultrasound scanner Vivid 7: Application SV 3.3.4, System SV 1.5.1 (2005.03.08).
}

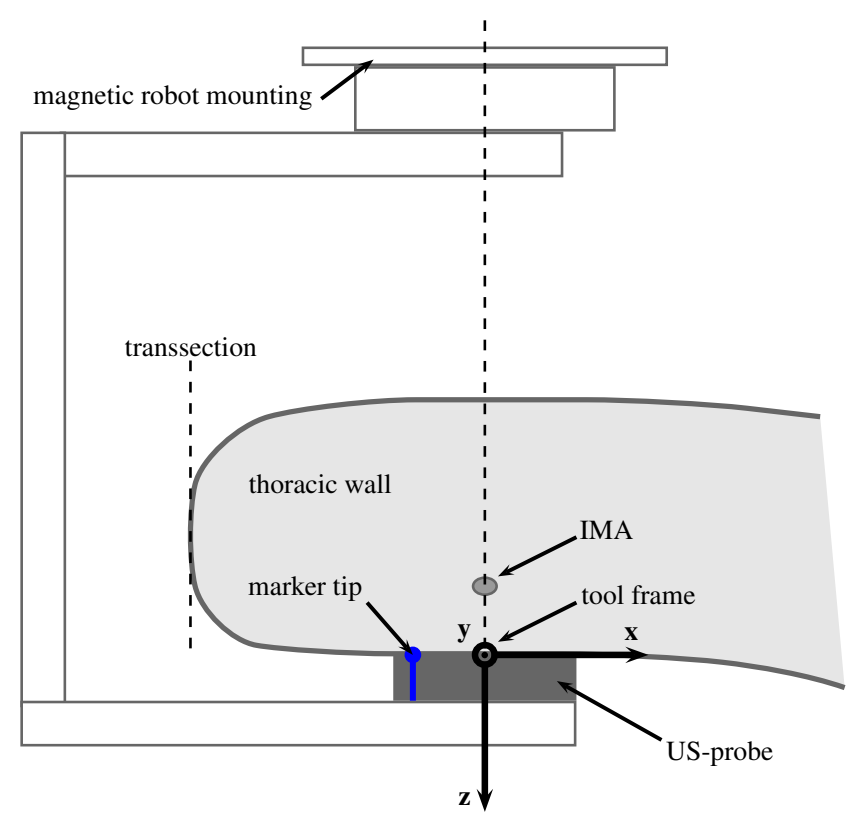

Fig. 2. C-shaped tool and US-probe mounting.

\section{OPERATION PROCESS}

The workflow of the introduced surgical intervention can coarsely be divided into three phases: 1) Probe positioning: The robot is moved to a predefined approach position outside of the patient by using the position control interface. Then it is switched to Cartesian impedance control, so the surgeon can manually move the tool to the position of the desired entry point for the IMA detection while checking the contact situation of the US-probe at the familiar ultrasonography images. 2) Artery detection: When the artery is visible and the feed axis (y-axis) of the tool is satisfyingly aligned with the transsection of the median sternotomy the robot is switched to the IMA detection mode. The MIRO robot guides the probe along the IMA and marks the path of the artery to the tissue surface. 3) Probe removal: The the probe is removed from the thorax using the impedance controlled mode. Afterward the surgeon is able to place his incision according to the detected path and can dissect the marked section of the IMA.

As mentioned above the probe is placed manually. The surgeon is in charge of initially aligning the $y$-axis parallel to the transsection and the $\mathrm{z}$-axis to the normal of the tissue surface. For the subsequent detection of the IMA the rotation of the probe is kept constant while the probe is moved along the inner side of the thoracic wall. This is possible as the physiological path of the IMA is fairly aligned with the transsection of the sternum and the curvature of the tissue surface is in a range that still allows Doppler US-imaging.

The system setup in the IMA detection mode is depicted in Fig. 3. The external interaction between the robotic system and the patient includes the effect of the robot to the tissue described by the probe velocity $\dot{x}$, the contact forces $f_{c}$ and the US-images $\mathbf{I}$.

The control input for the MIRO robot carrying the probe 
is the desired velocity $\dot{\mathbf{x}}_{\mathrm{d}}$. Additionally, the MIRO robot provides the measured external forces and torques $f_{\text {ext }}$ that are transformed to the probe frame. The position $\mathrm{x}$ of the artery with respect to the center of the probe is extracted by image processing from the US-images I that are also fed back to the surgeon. The desired velocity in y-direction $\dot{y}_{d}$ is directly adjusted by the surgeon. The depicted probe control includes the controllers to generate the desired velocity $\dot{\mathrm{x}}_{\mathrm{d}}$ from the measurements $x$ and $f_{\text {ext }}$ and the user input $\dot{y}_{d}$. For the detection of the IMA the position controlled mode of the MIRO robot is used. The translation of the probe is controlled separately for the three Cartesian axes of the tool frame introduced in Fig. 2.

The robot controller with high dynamics and the probe controller with lower dynamics are combined in a cascaded control scheme.

The constraints and the decoupled control schemes for the three tool axes are described in more detail in the following sections.

\section{A. Controller Constraints}

As the detection of the IMA is working without preoperatively gathered data, the controller is based on intraoperatively accessible data. Whereas the input of the surgeon and the force measurement are reliable input signals, the result of the image processing depends on the quality of the US-images. The inner side of the thoracic wall is a surface with unknown topology. The tissue below the IMA is not only soft tissue but also cartilage or alternating ribs and intercostal spaces. In addition, the whole thorax moves with the respiration and by forces induced by the tool to tissue contact. However, a good contact situation between the USprobe and the tissue surface is essential to obtain robust image processing results. Good contact in this context is equivalent to the entire surface of the probe being in contact at any time of the detection process. There are two adjustable factors in the system influencing the contact situation: The first one is the contact force between the US-probe and the tissue. On the one hand the force needs to be high enough to guarantee good tissue contact and on the other hand the contact should not cause too much tissue deformation

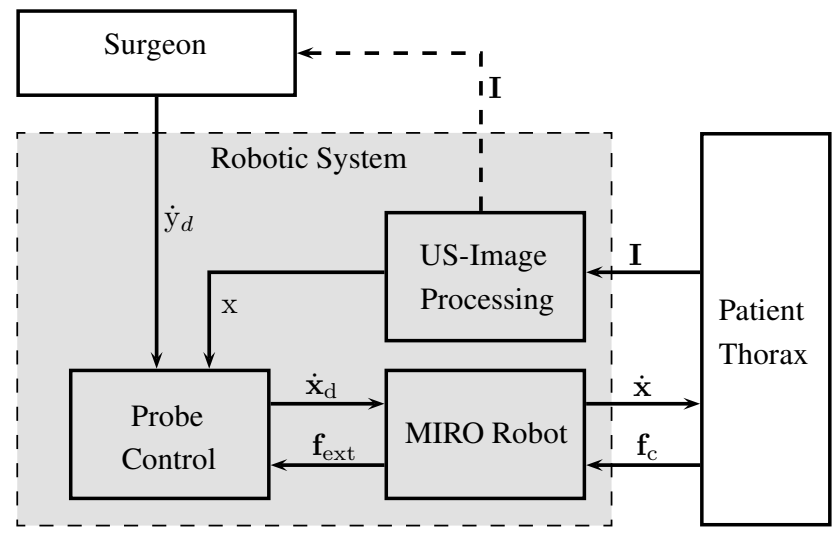

Fig. 3. System overview in IMA detection mode. so the detected path represents the real path of the artery. The second factor is the velocity of the tool. Decreasing the maximum velocity reduces the tracking behavior of the controller and therefore limits the system performance and accuracy. However, higher velocities result in extensive image disturbances in the Doppler US-images that are caused by the US-device misinterpreting the movements of the probe as flow inside the tissue or by partial and total contact losses. Additionally, the dynamics of the Cartesian controller is limited to achieve closed-loop stability with the cascaded controller structure. The stability of the controlled MIRO robot as inner loop is shown in [12]. The outer control loop is designed according to the Ziegler-Nichols tuning method to gain stability. Afterward the identified gains are reduced to match the constraints mentioned above.

As the setup aims for operations in realistic and preoperatively unknown environments the controller parameters are a trade-off between tracking performance and overall system robustness.

\section{B. Centering on Artery}

The $\mathrm{x}$-axis of the tool frame is in the plane of the tissue surface and approximately orthogonal to the artery direction. Therefore the control of the tool in the $\mathrm{x}$-direction is used to center the tool above the artery. The displacement of the artery is extracted from the US-images $\mathbf{I}$ by image processing. The controller is a heuristically adjusted PIDcontroller considering the constraints introduced above that additionally accomplishes the rate transition from the video frame rate of $25 \mathrm{~Hz}$ to the robot control rate of $3 \mathrm{kHz}$ by appropriate filtering. The desired velocity of the probe in the $\mathrm{x}$-axis is calculated from the input $\mathrm{x}$ additionally taking into account velocity limits and imaging quality.

As internal processing data in a standard ultrasound systems is not easily accessible the S-Video interface and image processing are used for the detection of the artery.

The first major sources of information in color Doppler

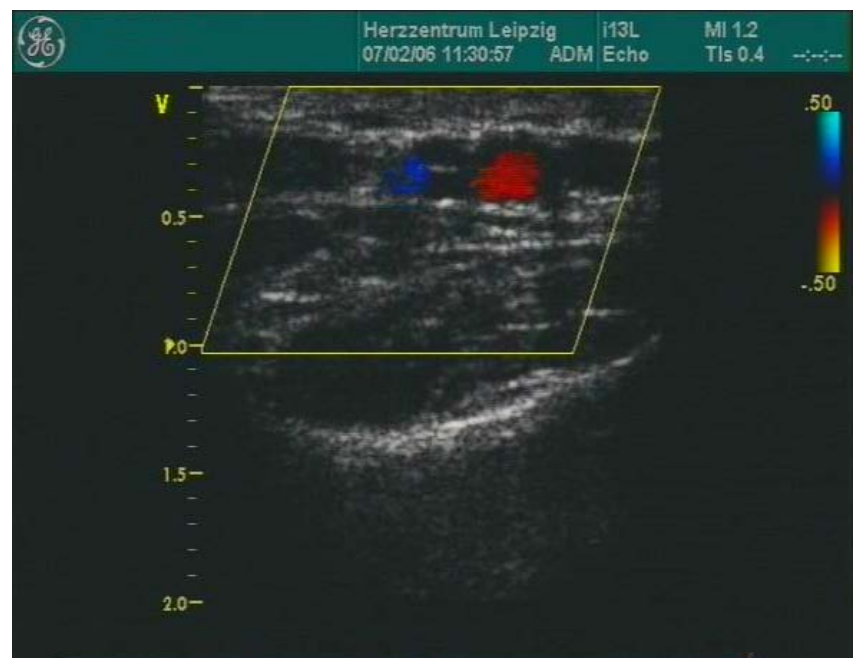

Fig. 4. Typical ultrasound image with color Doppler information of artery (red), vein (blue) and surrounding tissue. 
data are the colored pulsating appearance of arteries and the constant colored blobs indicating the veins in an otherwise black and white image as depicted in Fig. 4. In this case the artery is represented by a red blob and the vein by a blue one. The image characteristics are of course subject to adjustment of the US-system by the medical staff. For the image processing settings are chosen as to comply with the typical appearance for color Doppler applications.

As the images are grabbed in YUV color space a measure $\mathrm{S}$ similar to the image saturation can be calculated as follows:

$$
\mathrm{S}=\frac{\max (\mathrm{U}, \mathrm{V})-\min (\mathrm{U}, \mathrm{V})}{\min (\mathrm{U}, \mathrm{V})}
$$

Colored blobs with $\mathrm{S}>(128+$ threshold $)$ are selected according to morphological features such as compactness and area. Red and blue blobs can be distinguished by their mean gray value in the $\mathrm{U}$ and $\mathrm{V}$ images. The result of artery tracking in a video sequence solely based on this color blob information is depicted in Fig. 5 as red graph. The lateral position information can only be updated once per pulse and only if the pulse is detected with high quality in subsequent images. Branches of the artery can be neglected as they are usually smaller and are mainly directed to the tissue between the ribs. Using only this approach yields very low dynamics of the visual servoing loop as the maximum update rate is the frequency of the heartbeat.

A second source of information in the images is the lateral tissue movement in the B-mode signal of the ultrasound. This movement is apparent to the observer and can easily be analyzed by image processing. Using normalized cross correlation the best match of a template from $\mathbf{I}_{t-1}$ is found in $\mathbf{I}_{t}$. The resulting frame to frame position offset yields the trajectory depicted in Fig. 5 as green graph. Combining the color blob information and the B-mode lateral motion

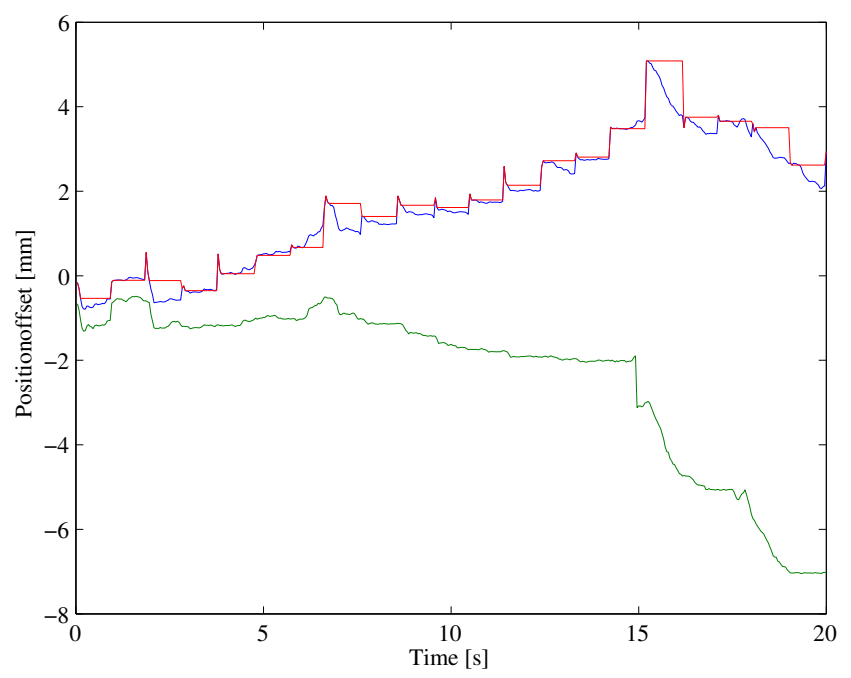

Fig. 5. Measured trajectory of the LIMA in an open loop video sequence. Red: color blob tracking, green: correlation based tracking, blue: combined tracking. results in the blue graph in Fig. 5. As can be seen the position hypothesis is updated by correlation when no pulse is visible and reset with each color blob detection. A confidence measure with a slow decay when no pulse is found and a fast decay when correlation fails, acts as quality criterion for the robot control. When tracking is lost the quality measure decays to zero and stops the robot. The tracking can restart with a position jump, when a sufficient number of pulses have been detected. The scaling between US-data and metric units is derived from the settings of the US-device.

The combination of the color blob detection and the correlation increases the update rate from the heartbeat frequency to the video frame rate of $25 \mathrm{~Hz}$ without interpolating or predicting the position of the artery.

\section{Feed Motion Control}

The velocity in the $y$-axis causes the feed motion in direction of the artery. The feed velocity can be influenced by the surgeon at any time so he is capable of reacting to critical situations like bigger branches of the artery or US-imaging quality loss. This approach adds the expert knowledge of the surgeon to the result of the detection run, whereas the surgeon intraoperatively gains knowledge about the path and depth of the artery by means of familiar US-imaging. Optionally the quality signal of the image processing is used to reduce the feed velocity with decreasing contact quality and stop at total US-image losses.

\section{Contact Force Control}

In Sec. III-A the need of good contact between US-probe and tissue is accentuated. To ensure the tissue contact the $\mathrm{z}$-axis of the tool frame aligned to the normal of the tissue surface is force controlled.

The controller for the $\mathrm{z}$-axis is a heuristically adjusted PD-controller considering the constraints mentioned. The controller input is the error between the measured force in $\mathrm{z}$-direction of the tool frame and the desired force that is a constant value. The controller output is the desired velocity of the probe in the z-axis.

The measurement of the forces is obtained without an additional force sensor. As the MIRO robot is equipped with torque sensors in every joint [11], the torques $\tau_{\text {ext }}$ caused by external forces can be calculated from the measured torques $\tau$ as proposed in [16] by solving

$$
\mathbf{M}(\mathbf{q}) \cdot \ddot{\mathbf{q}}+\mathbf{C}(\mathbf{q}, \dot{\mathbf{q}}) \cdot \dot{\mathbf{q}}+\mathbf{g}(\mathbf{q})=\tau+\tau_{\text {ext }},
$$

with the mass matrix $\mathbf{M}$, the influence of the Coriolis force $\mathbf{C}$ and the gravity compensation $\mathrm{g}$ depending on the joint angles $\mathbf{q}$ and their derivatives.

As the dynamics of the system are limited in any case the influence of the Coriolis force and of the mass matrix in the desired workspace are neglected. These assumptions lead to the simplified approximation for the external Cartesian forces $\mathbf{f}_{\text {ext }}$ by the formula

$$
\mathbf{f}_{\mathrm{ext}}=\mathbf{J}^{+T} \cdot(\tau-\mathbf{g}(\mathbf{q}))
$$


that is solved in every control cycle of the robot. Whereas, $\mathbf{J}^{+}$is the Moore-Penrose-Inverse of the Jacobian matrix, $\tau$ the vector of the measured torques and $\mathrm{g}$ the gravity compensation based on the vector of the motor sided joint angles q.

\section{EXPERIMENTAL RESULTS}

The setup introduced in Sec. II is developed and adjusted with a patient phantom and works well under laboratory conditions. However, as medical application it is important that the system with the MIRO robot and the attached USprobe also satisfies the needs of a surgeon during an in vivo intervention. The results presented in the following demonstrate the performance of the system in an animal trial with a living pig.

As described in Sec. III the tool is positioned by hand close to the cranial side of the artery using the impedance controlled MIRO robot in a way that the following initial conditions are satisfied: 1) The feed axis (y-axis of the tool frame) is approximately aligned to the transsection of the median sternotomy. 2) The z-axis of the tool frame is roughly the normal of the inner side of the thoracic wall. 3) The contact between US-probe and tissue is sufficient for USimaging. 4) The IMA is visible in the US-image.

In the following sections the evaluation of the experimental setup is described in three steps. All of the evaluation steps refer to the initial conditions introduced above.

\section{A. Force Control Evaluation}

In a first step the force control for the tool $\mathrm{z}$-axis is validated. As reference the force error is recorded in the initial tool position with deactivated force control. For further records the force control is activated. The control of the $\mathrm{x}$-axis and the $\mathrm{y}$-axis is deactivated. The desired contact force is set to $1 \mathrm{~N}$. For an easy comparison of the two cases the discrete Fourier transform of the error is used. The graphs in Fig. 6 are the discrete Fourier transforms of two recorded sequences of equal length. For the upper graph the force control is deactivated. As initially the tool is manually placed the contact force is different from the desired force. This constant force error causes the peak at zero $\mathrm{Hz}$ with an amplitude of about $0.03 \mathrm{~N}$. The following peaks are mainly attributed to the influence of respiration. As the pig is connected to a respirator the constant respiration frequency of about $0.34 \mathrm{~Hz}$ and several harmonics can be seen in the frequency domain. The influence of the heart beat at a frequency of about $1.1 \mathrm{~Hz}$ is scarcely visible. The lower graph results from activating the force controller. The constant component of the force error is eliminated as the transfer function of the velocity controlled MIRO robot includes an integral part. Additionally, the amplitude of the respiration frequency is reduced from about $0.06 \mathrm{~N}$ to $0.02 \mathrm{~N}$.

As mentioned in Sec. III-A it is essential to ensure a good contact situation. The residual force error is tolerated to avoid motion artifacts in the US-images and therefore achieve robustness. The recorded measurements demonstrate
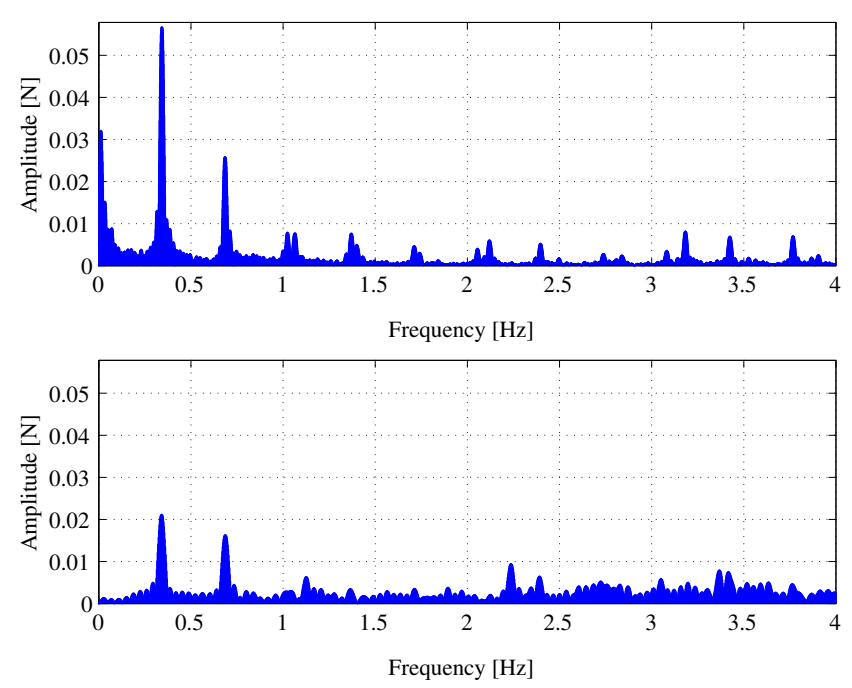

Fig. 6. Frequency analysis of contact force error. Top: deactivated controller. Bottom: activated force control.

that the residual force error reaches maximum values of about $\pm 0.2 \mathrm{~N}$ even with feed motion and visual servoing activated. Therefore the desired contact force of $1 \mathrm{~N}$ proves to be a reasonable choice as it provides good tissue contact while the blood flow through the artery is not disturbed and the displacement of the thoracic wall is far away from compromising the assumptions from Sec. III allowing the fixed tool rotation. Additional statistical data about the recorded sequences are given in Tab. I.

\section{B. IMA Detection Evaluation}

The second part of the experiments is the detection of the IMA. The experiments include detecting the left internal mammary artery (LIMA) as well as the right internal mammary artery (RIMA). In addition to the force controller the visual servoing loop controlling the $\mathrm{x}$-axis of the tool frame is activated. The feed velocity is set to $2 \frac{\mathrm{mm}}{\mathrm{s}}$ and can be switched or reversed as needed.

As the artery is visible in the US-image at the initial position the image processing measures the displacement of the artery according to the center of the probe. The controller output causes the MIRO robot to center the probe above the artery. As soon as the feed is switched on, the probe is moved across the tissue surface.

In the upper graph of Fig. 7 the probe position on the force controlled z-axis of the tool frame is illustrated. The probe follows the curvature of the inner side of the thoracic wall with the superimposed compensation for the thorax movement caused by respiration. The graph shows two interesting results: First, the system tolerates the curvature of the surface. Along the detected section of about $6 \mathrm{~cm}$ the z-position alters by $6 \mathrm{~mm}$ and still a robust detection is achieved. Second, the amplitude of the respiration rises with increasing distance to the initial position that is located at the cranial side of the transsection. The lower graph depicts the error of the contact force that also increases with higher 

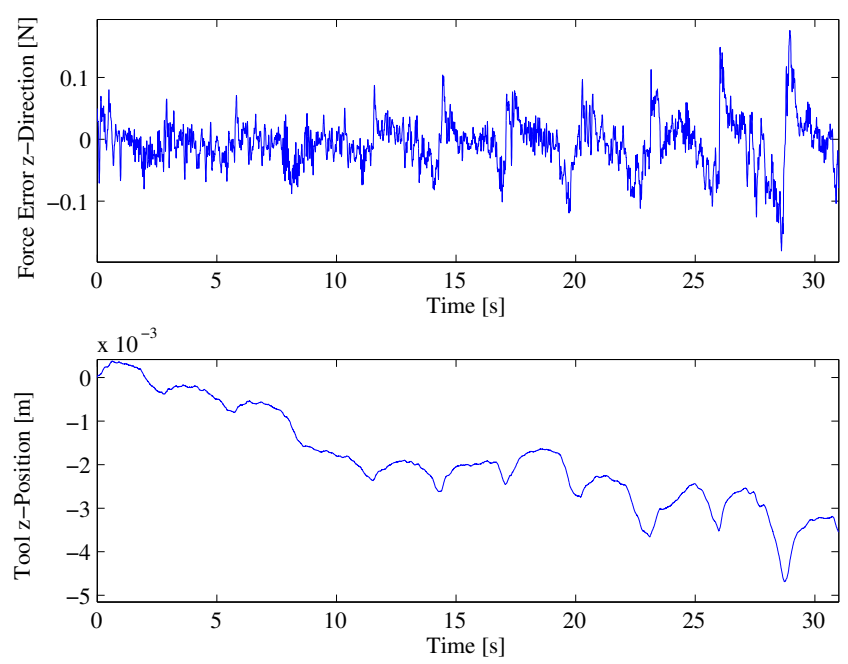

Fig. 7. Tool position and force error in z-axis with feed motion.

respiration influence. Statistical data about the force error are given in Tab. I.

In Fig. 8 the detected path of the LIMA is depicted. The smooth blue line is the path of the probe center in the $x-y-$ plane of the tool frame. The red line is the path of the artery detected by image processing. This path is composed of the path of the tool center and the result of the image processing with a mean value $\bar{x}$ of $0.1 \mathrm{~mm}$, a standard deviation $\sigma(x)$ of $0.6 \mathrm{~mm}$ and an amplitude between $-1.7 \mathrm{~mm}$ and $1.5 \mathrm{~mm}$. The $\mathrm{x}$-axis of the graph is the relative y-position of the tool according to the initial position and is aligned to the transsection of the median sternotomy. The detected section of the artery spans a length of about $6 \mathrm{~cm}$. The y-axis is the position of the probe in the $\mathrm{x}$-axis of the tool frame that is controlled by visual servoing. Repeated detection cycles supply similar detection quality. With the surgeon controlling the feed, the artery is robustly detected within about $30 \mathrm{~s}$.

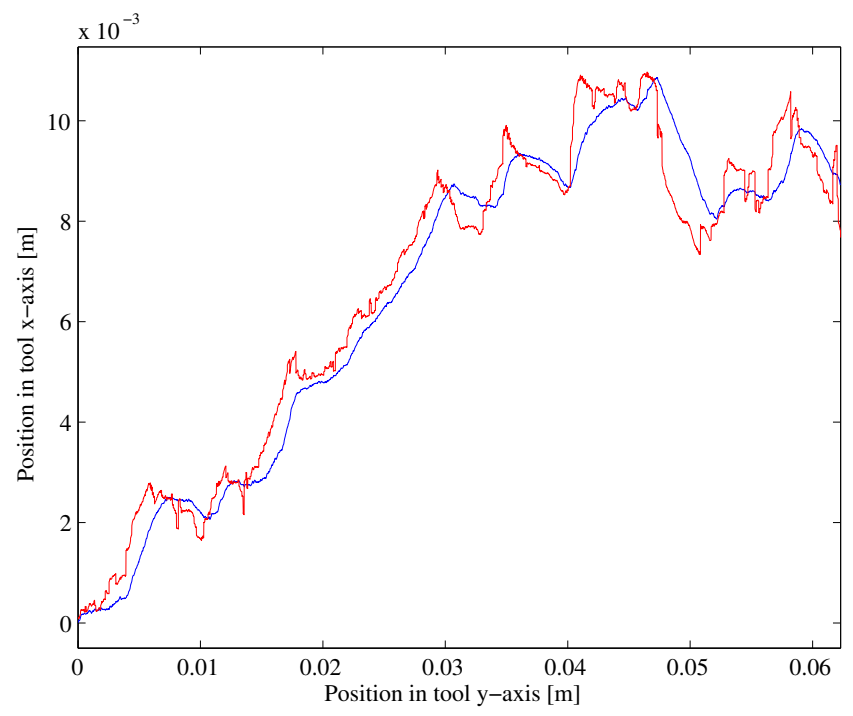

Fig. 8. Detected path of the LIMA in the $x-y-p l a n e$ of the tool frame.
TABLE I

STATISTICS OF FORCE ERROR MEASUREMENTS.

\begin{tabular}{c|c|c|c|c} 
& $\overline{\mathrm{f}}_{\mathrm{e}}[\mathrm{N}]$ & $\sigma\left(\mathrm{f}_{\mathrm{e}}\right)[\mathrm{N}]$ & $\min \left(\mathrm{f}_{\mathrm{e}}\right)[\mathrm{N}]$ & $\max \left(\mathrm{f}_{\mathrm{e}}\right)[\mathrm{N}]$ \\
\hline no controller & -1.0873 & 0.0537 & -1.2469 & -0.9661 \\
\hline force control & -0.0007 & 0.0345 & -0.2225 & 0.2048 \\
\hline detection run & -0.0061 & 0.0394 & -0.1804 & 0.1759
\end{tabular}

The contouring error between the detected path and the path of the probe in Fig. 8 is ascribed to the limited tracking behavior of the controller. As introduced in Sec. III-A the need for disturbance-free US-imaging constricts the velocity of the probe. However, because of the resulting lowpass characteristics of the controlled system the IMA detection tolerates the influence of small branches that may disturb the detection of the artery center.

\section{Validation of the Marked IMA Path}

The third experimental step is the validation of the marked IMA path. The marker is mounted to the tool so the detected path is drawn to the tissue surface. The displacement of the marker related to the center of the probe is larger than the maximum expected error of $\pm 2 \mathrm{~mm}$. This ensures that the marked trajectory is save for cutting without injuring the artery.

Then the path of the LIMA is marked in the cycle introduced in Fig. 8 taking about half a minute. Afterward the surgeon cuts along the marked path with an electrocauter. The artery is not injured because of the residual distance between the marked path and the artery. To show that the marked path is close to the real path of the artery the surrounding tissue is carefully removed. The thickness of the removed tissue at the chosen marker distance is about $2 \mathrm{~mm}$ along the marked path. From this follows that the system is capable of marking a secure path for dissecting the IMA. When the artery is used as bypass graft it is dissected with some supporting tissue around the artery. In this context the residual layer of tissue is an important feature and the thickness of the layer can be influenced by the position of the marker. Besides, the measured peak detection error can be tolerated in the surgical intervention.

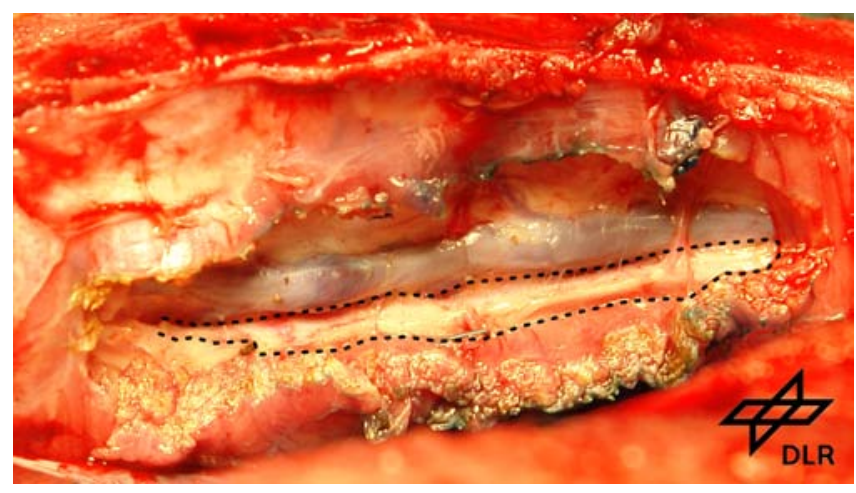

Fig. 9. Dissected RIMA.

The same process is then successfully repeated for the 
RIMA. In Fig. 9 the result after cutting the marked path and removing the residual tissue is shown. The RIMA (surrounded by the dotted line) is not injured and could be used as coronary artery bypass. The initial position of the detection is at the right side of the image. Remains of the marker color are visible at the borders of the incision.

\section{CONCLUSIONS AND FUTURE WORKS}

\section{A. Conclusions}

The surgical lightweight robot MIRO is used to guide a Doppler US-probe along the path of the left (LIMA) or right internal mammary artery (RIMA). A marker attached to the tool draws the path to the inner side of the thoracic wall so the surgeon can easily dissect the artery without injuring it. The robot therefore is controlled with a hybrid control scheme. The $\mathrm{x}$-axis of the tool is orthogonal to the artery and controlled by visual servoing on Doppler US-images. The position of the artery extracted from the images is used to center the probe above the artery. The y-axis of the tool is aligned to the artery. The velocity on this axis is directly controlled by the surgeon and causes the feed along the path of the IMA. The z-axis of the tool that is normal to the tissue surface is force controlled to ensure good contact between tissue and US-probe in spite of the preoperatively unknown surface topology and respiration movements of the thorax. The measurement of the contact forces is based on the internal torque sensors of the MIRO robot. However, the need for disturbance-free US-imaging limits the velocity of the probe and therefore the tracking behavior of the applied hybrid control. Anyhow, the system performs well in detecting the IMA with an accuracy that is adequate for the aimed intervention. The setup is validated in an animal trial with a pig where the LIMA and RIMA are marked and afterward dissected by the surgeon without injuring the arteries.

\section{B. Future Works}

The setup described in this paper is dedicated to assisting in conventional open chested surgical interventions. As it is foreseeable that the amount of endoscopic interventions will increase with improving technical possibilities, a similar system for these endoscopic operations has to be designed. The hybrid control presented in III can easily be adapted. Yet, the tool needs to be redesigned. Instead of the simple c-shaped tool, an instrument is needed that consists of a USprobe at the distal end, a force sensor close to the probe and an additional 2 DOF joint inside the body to regain the degrees of freedom lost because of the trocar point.

An other enhancement would be attaching the electric cauter directly to the tool replacing the marker. The surgeon would then control the feed velocity and switch the cautering. The most significant questions are how to prevent injury to surrounding vessels and nerves and how to handle branches. In this scenario the depth of the artery inside the tissue also has to be taken into account. Fortunately, the depth information is already contained in the US-images.

\section{ACKNOWLEDGMENTS}

The authors gratefully acknowledge the DFG for funding the research within the scope of the project "Development of a semi-automatic surgical assistance system for harvesting the internal thoracic artery based on intraoperative imaging and feedback control.", Prof. Dr. V. Falk and Dr. S. Jacobs at the ETH Zurich for the organization and accomplishment of the animal trial and W. Korb at the ICCAS, University of Leipzig, for providing the patient phantom.

\section{REFERENCES}

[1] Arthur M. Vineberg and Beverly L. Jewett, Anastomosis Between Coronary Vessels And Internal Mammary Artery, Canadian Medical Association Journal, vol. 56(6), 1947, pp. 609-614.

[2] B. Kiaii, W. D. Boyd, R. Rayman, W. B. Dobkowski, S. Ganapathy, G. Jablonsky and R. J. Novick, Robot-Assisted Computer Enhanced Closed-Chest Coronary Surgery: Preliminary Experience Using a Harmonic Scalpel $\left(\right.$ and ZEUS $^{\mathrm{TM}}$, Minimally Invasive Cardiac Surgery (MICS) Symposium, 2000.

[3] Chieh-Sung Tsai, Chuan-Nan Su, Chih-Yuan Lin, Gou-Jieng Hong, Guang-Huan Sun, Dah-Shyong Yu and Shih-Hurng Loh, Early Experience of Robotic-Assisted Coronary Artery Bypass Grafting, Acta Cardiol Sin, vol. 22, 2006, pp. 154-158.

[4] Stephan Jacobs and Volkmar Falk, Medical Robotics in Cardiac Surgery, Medical Robotics, I-Tech Education and Publishing, Vienna, Austria, ISBN 978-3-902613-18-9, 2008, pp. 167-174.

[5] P. Nataf, N. Al-Attar, R. Ramadan, M. Scorcin, R. Raffoul, S. Salvi and A. Lessana, Thoracoscopic IMA takedown, Journal of Cardiac Surgery, vol. 15(4), 2000, pp. 278-282.

[6] Armin Oehlinger, Nikolaos Bonaros, Thomas Schachner, Elisabeth Ruetzler, Guy Friedrich, Guenther Laufer and Johannes Bonatti, Robotic Endoscopic Left Internal Mammary Artery Harvesting: What Have We Learned After 100 Cases?, The Annals of Thoracic Surgery, vol. 83(3), 2007, pp. 1030-1034.

[7] Thomas A. Vassiliades, Technical Aids to Performing Thoracoscopic Robotically-Assisted Internal Mammary Artery Harvesting, The Heart Surgery Forum, vol. 5(2), 2002, pp. 119-124.

[8] R. A. Beasley and R. D. Howe, Tactile tracking of arteries in robotic surgery, Proceedings of the IEEE International Conference on Robotics and Automation (ICRA), vol. 4, 2002, pp. 3801-3806.

[9] Wilhelm Schberle, Ultrasonography in Vascular Diagnosis, SpringerVerlag Berlin Heidelberg, ISBN 3-540-23220-6, 2005.

[10] Eric C. Pua, Matthew P. Fronheiser, Joanna R. Noble, Edward D. Light, Patrick D. Wolf, Daniel von Allmen and Stephen W. Smith, 3-D Ultrasound Guidance of Surgical Robotics: A Feasibility Study, IEEE Transactions on Ultrasonics, Ferroelectrics, and Frequency Control, vol. 53(11), 2006.

[11] U. Hagn, M. Nickl, S. Jörg, G. Passig, T. Bahls, A. Nothhelfer, F. Hacker, L. Le-Tien, A. Albu-Schffer, R. Konietschke, M. Grebenstein, R. Warpup, R. Haslinger, M. Frommberger and G. Hirzinger, The DLR MIRO: A versatile lightweight robot for surgical applications, Industrial Robot: An International Journal 2008, vol. 35(4), 2008, pp. 324-336.

[12] L. Le-Tien, A. Albu-Schäffer and G. Hirzinger, MIMO State Feedback Controller for a Flexible Joint Robot with Strong Joint Coupling, Proceedings of the IEEE International Conference on Robotics and Automation (ICRA), 2007, pp. 3824-3830.

[13] L. Le-Tien, A. Albu-Schäffer, A. De Luca and G. Hirzinger, Friction observer and compensation for control of robots with joint torque measurement, IROS, 2008, pp. 3789-3795.

[14] Ch. Ott, A. Albu-Schäffer, A. Kugi, S. Stramigioli and G. Hirzinger, A Passivity Based Cartesian Impedance Controller - Part I: Torque Feedback and Gravity Compensation, Proceedings of the IEEE International Conference on Robotics and Automation (ICRA), 2004, pp. 2659-2665.

[15] A. Albu-Schäffer, Ch. Ott and G. Hirzinger, A Passivity Based Cartesian Impedance Controller - Part II: Full State Feedback, Impedance Design, and Experiments, Proceedings of the IEEE International Conference on Robotics and Automation, 2004, pp. 2666-2672.

[16] M. Spong, Modeling and control of elastic joint robots, IEEE Journal of Robotics and Automation, vol. 3, 1987, pp. 291300. 